\title{
First reported case of transfusion-transmitted Ross River virus infection
}

\author{
We describe the first documented case of Ross River virus (RRV) infection transmitted by blood \\ transfusion. The recipient had a clinically compatible illness, and RRV infection was confirmed by \\ serological tests. The implicated donation was positive for RRV RNA. We discuss the risk to blood \\ recipients and the implications for blood donation in Australia.
}

\section{Clinical record}

In May 2014, the Australian Red Cross Blood Service (the Blood Service) in Western Australia received a delayed notification from a donor who had developed fatigue and arthralgia 2 days after giving blood in March 2014 and was subsequently diagnosed with acute Ross River virus (RRV) infection (Box).

Veronica C Hoad MBBS, MPH, FAFPHM

David J Speers FRACP, FRCPA, FACTM²

Anthony J Keller FRACP, FRCP, MRCPath

Gary K Dowse MSC, DTM\&H, FAFPHM

Clive R Seed $\mathrm{BSc}, \mathrm{PhD}^{\prime}$

\section{Michael D A Lindsay}

$\mathrm{BSC}, \mathrm{PhD}^{3}$

Helen M Faddy BSc(Hons), PhD ${ }^{4}$

Joanne Pink FRACP, FRCPA, GAICD

1 Australian Red Cross Blood Service, Perth, WA.

2 PathWest Laboratory Medicine WA Perth, WA

3 Department of Health Western Australia Perth, WA.

4 Australian Red Cross Blood Service, Brisbane, QLD.

vhoad@

redcrossblood.org.au

doi: 10.5694/mjal4.01522
PathWest Laboratory Medicine WA detected RRV IgM antibodies using an inhouse indirect immunofluorescence antibody (IFA) test, but no RRV antibodies were detected using an inhouse haemagglutination inhibition (HI) antibody test 10 days after blood donation. RRV IgM antibodies are detected by IFA testing within a few days of onset of illness and routinely persist for several weeks or, occasionally, months or years. IFA tests are less prone to false-positive results compared with enzyme immunoassays. The HI antibody test primarily detects IgG antibodies, which appear within several weeks but after the IgM response.

Blood Service procedure stipulates that donors with a diagnosed RRV infection are unable to donate fresh components for 4 weeks after recovery. Moreover, fresh components donated from 4 weeks before illness onset to 4 weeks after recovery must be recalled.

The components from the implicated donation were identified: the red blood cell (RBC) component had been transfused to a patient on 12 March 2014, the plasma had been pooled for the manufacture of plasma-derived products and the platelet component had not been used. The treating clinician of the $\mathrm{RBC}$ recipient was notified as part of the recall procedure.

The recipient was having regular blood transfusions due to myelodysplastic syndrome that was associated with chronic fatigue and joint pains. The recipient reported a worsening of symptoms in the months after transfusion of the infected blood; however, there was not a clear exacerbation of these symptoms consistent with the incubation period of RRV.

\section{"the Blood Service is taking steps to}

\section{strengthen its messaging to donors regarding} development of post-donation illnesses"

On notification from the Blood Service, the recipient's treating clinician requested serological testing for RRV on 28 May 2014, which found detectable IgM antibodies using the IFA assay and a high titre of antibodies by HI testing (antibody titre, $>1: 640$ ). The detection of both $\mathrm{IgM}$ and $\mathrm{HI}$ antibodies indicates RRV infection in recent months. Previous testing for RRV IgM and HI antibodies in 2006 and August 2013 had been negative. Subsequent inhouse reverse transcriptase polymerase chain reaction (RT-PCR) analysis for RRV RNA performed on stored serum from 28 May gave a negative result. These results are consistent with RRV illness several months before 28 May, with resolution of the transient viraemic phase. No samples from the recipient in March 2014 were available for serological or PCR testing.

In response to this possible case of transfusion-transmitted RRV, the associated archived donor sample was retrieved and sent to the Victorian Infectious Diseases Reference Laboratory for RRV serological tests and RT-PCR analysis. This sample tested negative for RRV IgM and IgG but RRV RNA was detected by two inhouse RT-PCR tests and verified by sequencing. These results are consistent with the blood donation being collected

Timeline of major events related to the case of Ross River virus (RRV) transfusion transmission, 2014

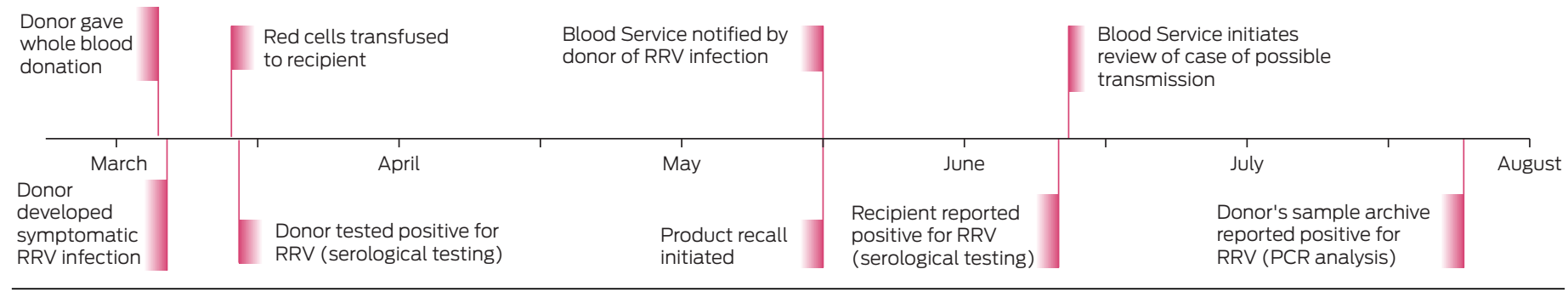

$\mathrm{PCR}=$ polymerase chain reaction. 
during the pre-seroconversion but transient viraemic phase of RRV illness.

\section{Discussion}

Since the isolation of RRV from humans was first reported in 1972, ${ }^{1}$ our understanding of the epidemiology of the disease has increased considerably. RRV is now known to be the most common mosquito-borne disease of humans in Australia, ${ }^{2}$ and is endemic in several regions. An average of around 5000 cases of RRV disease are notified annually in Australia, with considerable yearly, seasonal and regional variability due to differences in environmental conditions that affect the mosquito vectors and native animal hosts of the virus. ${ }^{2}$ The incubation period averages 7 to 9 days with a range of 2 to 21 days. ${ }^{3}$ Symptoms of RRV most commonly include joint manifestations, which are usually symmetrical and acute in onset, with rash and fever being less common. ${ }^{3}$ As many as 55\% to $75 \%$ of RRV infections are asymptomatic. ${ }^{3}$

Arboviruses such as dengue viruses and West Nile virus are known to be transfusion transmissible, ${ }^{4}$ and the potential of RRV to be transfusion transmissible was raised in this Journal in $1995 .^{5}$

Although not previously documented, transfusion transmission of RRV has been considered theoretically possible, given a likely period of asymptomatic viraemia before the onset of symptoms. ${ }^{4,6}$ This risk is supported by the observation of asymptomatic viraemia typically lasting 5 days after RRV infection in a mouse model. ${ }^{7}$ On the assumption that transfused blood could transmit RRV, this study estimated the risk of RRV transfusion transmission during a 2004 outbreak in Cairns as one in 13542 donations. This risk was of the same order of magnitude as that estimated for dengue virus transmission by transfusion during a contemporaneous dengue fever outbreak in Cairns. ${ }^{7}$

The donor we describe developed an illness clinically compatible with RRV infection 2 days after donating blood and was shown to have a serological profile consistent with acute RRV infection 10 days after donating. The donated blood was subsequently shown to contain RRV RNA by two inhouse RT-PCR tests, and this was confirmed by sequencing.

While the exacerbation of the chronically ill recipient's fatigue and muscle and joint pains was not clearly consistent with the incubation period of RRV subsequent to the transfusion, the results of RRV serological tests performed about 2 months after transfusion were consistent with infection within this 2-month period. Unfortunately, there were no stored blood specimens collected from the recipient shortly after receiving the blood donation, and hence it was not possible to compare sequences with the donor virus to confirm transmission.

Surveillance by the WA Department of Health showed that the recipient was the only person for whom RRV infection was reported between 1 July 2013 and 30 June 2014 from the local government area in which she resided. The recipient also spent most of her time indoors and could not recall being bitten by mosquitoes. Taken together, these lines of evidence strongly support the likelihood that the recipient's RRV infection was transmitted by transfusion. Thus, this is the first report of transfusion-transmitted RRV.

Laboratory testing for RRV is not done for Australian blood donors during the donation process, and there is no validated blood screening test for RRV. To manage the risk of transfusion transmission, the Blood Service does not permit donors with symptoms compatible with RRV to donate until they are fully recovered. However, given that most RRV infections are asymptomatic and viraemia is present during the incubation period, excluding donors based on symptoms will not prevent all potentially infectious donations entering the blood supply. Provided infected donors report subsequent illness immediately to the Blood Service, the recall process should prevent the proportion of donations from symptomatic RRV infected donors from being used. Unfortunately, in this case, where notification was delayed for 2 months, the blood component had already been transfused. In response to this, the Blood Service is taking steps to strengthen its messaging to donors regarding development of postdonation illnesses.

In 2012, the Blood Service established a sample archive of every blood donation to meet regulatory standards and assist in investigation and lookback (tracing and notifying patients who may have received infected blood components and investigating donations and donors when a patient has a suspected transfusion-transmissible infection). This archive provides the ability to perform further testing on samples from past donations, as in this case, providing data on the actual risk associated with transfused donations from implicated donors and for investigations where an infection is reported in a recipient.

Transfusion transmission of RRV no longer appears to be only a theoretical risk. However, with about 5000 mosquito-related RRV notifications per year, transfusion transmission of RRV - or the related Barmah Forest virus, which has a lower incidence - is likely to remain a rare event. Any actions taken to prevent infected components entering the blood supply need to take into account the cost, the impact on supply and the severity of the infection in recipients. Laboratory screening is not a feasible option, given that RRV nucleic acid testing is not validated for blood donation screening or available for the large-scale nucleic acid detection equipment used by the Blood Service. In addition, the cost of individual testing is unlikely to be cost-effective and, although RRV can cause debilitating symptoms in some patients, most infections are either asymptomatic or mild and self-limiting. ${ }^{8}$

Identifying donors who are at risk of exposure and temporarily excluding them from donating fresh blood components in areas and times of RRV outbreaks is one potential risk-mitigation option. When this strategy was applied to dengue fever, it was estimated to cost the Blood Service around \$1.0-\$3.8 million. ${ }^{9}$ However, irrespective 
of the financial cost, this option is unlikely to be feasible, since RRV is endemic in many parts of Australia and such restrictions might have a critical impact on supply. Pathogen reduction technology (PRT) is an alternative risk-management option that would not have an impact on supply. The Blood Service is investigating the effectiveness of PRT for the prevention of arboviral transfusion transmission, including RRV, but further research is needed..$^{10}$

The Australian blood supply is one of the safest in the world with respect to transfusion-transmitted infections. Yet, it is important to remember that blood transfusion is not without risk and should only be undertaken when the efficacy of the transfusion and improved clinical outcome outweigh the risks. ${ }^{11}$

Acknowledgements: We thank the recipient, the donor and the recipient's doctors for their input and permission to publish this information; and the Medical Service staff at the Blood Service and the Public Health Unit staff who assisted with following up the recipient. Australian governments fund the Blood Service to provide blood, blood products and services to the Australian community.

Competing interests: No relevant disclosures.

Received 30 Oct 2014, accepted 12 Feb 2015.

References are available online at www.mja.com.au. 
1 Doherty RL, Carley JG, Best JC. Isolation of Ross River virus from man. Med J Aust 1972; 1: 1083-1084.

2 Knope KE, Doggett SL, Kurucz N, et al. Arboviral diseases and malaria in Australia, 2011-2012: annual report of the National Arbovirus and Malaria Advisory Committee. CDI 2014; 38 : E122-E142.

3 Harley D, Sleigh A, Ritchie S. Ross River virus transmission, infection and disease: a cross-disciplinary review. Clin Microbiol Rev 2001; 14: 909-932.

4 Petersen LR, Busch MP. Transfusion-transmitted arboviruses. Vox Sang 2010; 98: 495-503.

5 Weinstein P, Weinstein SR, Rowe RJ. Transfusions: how many cases of Ross River virus infection do we cause? Med J Aust 1995; 163: 276.

6 Dunstan RA, Seed CR, Keller AJ. Emerging viral threats to the Australian blood supply. Aust N Z J Public Health 2008; 32: 354-360.

7 Shang G, Seed CR, Gahan ME, et al. Duration of Ross River viraemia in a mouse model - implications for transfusion transmission. Vox Sang 2012; 102: 185-192.

8 Harley D, Bossingham D, Purdie DM, et al. Ross River virus disease in tropical Queensland: evolution of rheumatic manifestations in an inception cohort followed for six months. Med J Aust 2002; 177: 352-355.

9 Faddy HM, Seed CR, Fryk JJ, et al. Implications of dengue outbreaks for blood supply, Australia. Emerg Infect Dis 2013; 19: 787-789.

10 Faddy HM, Prow NA, Fryk JJ, et al. The effect of riboflavin and ultraviolet light on the infectivity of arboviruses. Transfusion 2014; Nov 5 [Epub ahead of print].

1 National Blood Authority. Patient blood management guidelines: module 3-Medical. Canberra: NBA, 2012. http:// www.blood.gov.au/pbm-module-3 (accessed Aug 2014). 